Supporting Information

\title{
Flexible Piezoelectricity of Two-Dimensional Materials Governed by Effective Berry Curvature
}

Yilimiranmu Rouzhahong, Chao Liang, Chong Li, Huashan Li* and Biao Wang*

School of Physics, Sun Yat-sen University, Guangzhou 510275, China

E-mail: lihsh25@mail.sysu.edu.cn

E-mail: wangbiao@mail.sysu.edu.cn 


\section{A. Out-of-plane piezoelectricity}

For out-of-plane direction, periodic boundary condition is not fulfilled, and thus the gaugeinvariant phase of Bloch wave function is not a legitimate variable. This means both the berry curvature and pseudomagnetic field are not well defined. As a result, the analytical model developed in this study for predicting the in-plane piezoelectricity is not applicable to describe the out-of-plane piezoelectricity. Alternatively, considering that the polarization of finite system can safely adopt the classical definition, the piezoelectricity of monolayers along the out-of-plane direction can be calculated via the expectation value of position operator. With such definition, we can no longer take advantage of the simplification to treat strain induced electronic polarization by integral of berry curvature, and instead need to directly investigate the charge density redistribution under strain, which is beyond the scope of this work.

\section{B. Two-band tight binding Hamiltonian}

As illustrated in Figure S1, the variables associated with the two-band tight binding Hamiltonians include on site energy $\triangle$, nearest neighbor hopping energy $t_{i}$, axis distance between adjacent atoms $x_{0}$, bond length $a_{1}=a_{2}=l$, lattice vector $a$ and $b$. In this study, only the three equivalent nearest neighbor hopping $t_{1}=t_{2}=t_{3}$ is considered for buckled and planer structures, while both the nearest neighbor hopping $t_{1}$ and next nearest neighbor hopping $t_{2}=t_{3}$ are considered for puckered structures. The tight binding Hamiltonian takes the form as

$$
\boldsymbol{H}_{0}=\sum_{i} \sigma_{i} f_{i}(\boldsymbol{k})
$$


where $\sigma_{i}$ represents the Pauli matrices,

$$
\begin{gathered}
\left.f_{x}(\boldsymbol{k})+i f_{y}(\boldsymbol{k})=-t_{1} e^{-i x_{0} \cdot \boldsymbol{k}}-t_{2}\left(\mathrm{e}^{i \boldsymbol{k} \cdot \boldsymbol{a}_{1}}+\mathrm{e}^{i \boldsymbol{k} \cdot \boldsymbol{a}_{2}}\right)\right] \\
f_{z}(\boldsymbol{k})=\triangle .
\end{gathered}
$$

The two-band tight binding Hamiltonian for unstrained TCH monolayers is therefore written as

$$
\boldsymbol{H}_{0}=\left(\begin{array}{cc}
\Delta & \delta+\alpha_{x} k_{x}{ }^{2}+\alpha_{y} k_{y}{ }^{2}-i v k_{x} \\
\delta+\alpha_{x} k_{x}{ }^{2}+\alpha_{y} k_{y}{ }^{2}+i v k_{x} & -\Delta
\end{array}\right)
$$

with the parameters defined as

$$
\begin{gathered}
\delta=\mathrm{t}_{1}-2 \mathrm{t}_{2} \\
v=-2 a_{x} \mathrm{t}_{2}-\mathrm{t}_{1} x_{0} \\
\alpha_{x}=\mathrm{t}_{2} a_{x}{ }^{2}-\mathrm{t}_{1} x_{0}{ }^{2} / 2 \\
\alpha_{y}=\mathrm{t}_{2} a_{y}{ }^{2}
\end{gathered}
$$

where $a_{x}\left(a_{y}\right)$ represents the bond length projection on $x(y)$ axis. 
(a)

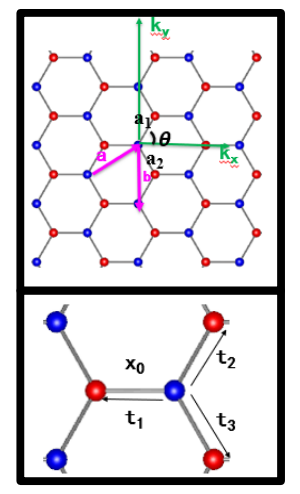

(b)

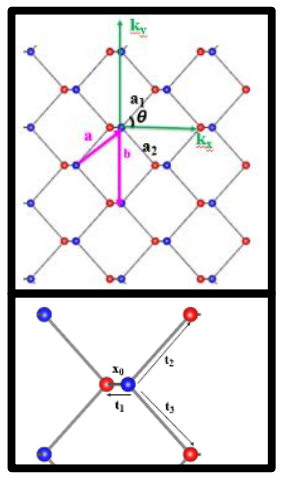

(c)

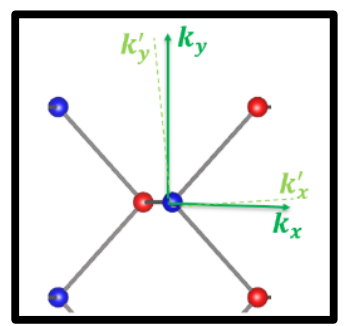

Figure S1. Illustration of two-band Hamiltonian for TCH monolayers. Variable definitions of

(a) buckled/planer structure and (b) puckered structure. Schematic change of puckered structure in reciprocal space under strain (c).

\section{Strained Hamiltonian}

Strain is described by a perturbation term in tight binding Hamiltonian ${ }^{1,2}$ (Figure S1 c) in this study, which takes the form as

$$
f_{\xi}^{\prime}=t_{1}^{\prime}+t_{2}^{\prime} \cos \theta+t_{3}^{\prime} \cos \theta-i\left(t_{2}^{\prime}-t_{3}^{\prime}\right) \sin \theta
$$

The nearest neighbor hopping of strained system is derived from that of pristine system as

$$
\begin{gathered}
t_{1}^{\prime}=-\beta t_{1} \varepsilon_{x x} \\
t_{2}^{\prime}=-\beta t_{2} \sin ^{2} \theta\left(\varepsilon_{x x} \cot ^{2} \theta+2 \varepsilon_{x y} \cot \theta+\varepsilon_{y y}\right) \\
t_{3}^{\prime}=-\beta t_{2} \sin ^{2} \theta\left(\varepsilon_{x x} \cot ^{2} \theta-2 \varepsilon_{x y} \cot \theta+\varepsilon_{y y}\right) .
\end{gathered}
$$

where $\varepsilon_{i j}$ represents the strain.

The pseudomagnetic vector potential $A_{x}\left(A_{y}\right)$ in Hamiltonian is simplified as 


$$
\begin{gathered}
A_{x}=-\beta\left(t_{1} \varepsilon_{x x}+2 t_{2}\left(\varepsilon_{x x} \cos ^{3} \theta+\varepsilon_{y y} \sin ^{2} \theta \cos \theta\right)\right) \\
A_{y}=-4 \beta t_{2} \varepsilon_{x y} \sin ^{2} \theta \cos \theta
\end{gathered}
$$

\section{Simplification of piezoelectric coefficient}

To simplify the piezoelectric coefficient $\mathrm{e}_{x x}$ in Eq. 9 of main article,

$$
e_{x x}=-\frac{4 \mathrm{e}}{(2 \pi)^{2}} \int \frac{v \alpha \beta \Delta}{4\left(\left(\delta+\alpha_{x} k_{x}^{2}+\alpha_{y} k_{y}^{2}\right)^{2}+\left(v k_{x}\right)^{2}+\Delta^{2}\right)^{3 / 2}} d \boldsymbol{k}
$$

we implement a scaling transformation, $q_{x} \equiv \sqrt{\alpha_{x}} k_{x}, \quad q_{y} \equiv \sqrt{\alpha_{y}} k_{y} \quad$ and $S_{B Z, q}=\pi q^{2}=\sqrt{\alpha_{x} \alpha_{y}} S_{B Z}$, where $S_{B Z}$ is the first Brillouin zone (BZ) area. For buckled, planer and puckered structures, $S_{B Z}$ can be simplified as $S_{B Z}=\frac{4 \pi^{2}}{l_{x} l_{y}}=\frac{c}{x_{0}{ }^{2}}$. The integral can be rewritten as

$$
e_{x x}=-\frac{4 e v \alpha \beta \Delta}{\sqrt{\alpha_{x} \alpha_{y}}(2 \pi)^{2}} \int \frac{d \boldsymbol{q}}{4\left(\left(\delta+q_{x}^{2}+q_{y}^{2}\right)^{2}+\left(v q_{x}\right)^{2} / \alpha_{x}+\Delta^{2}\right)^{3 / 2}}
$$

Use the polar coordinates,

$$
e_{x x}=-\frac{4 e v \alpha \beta \Delta}{\sqrt{\alpha_{x} \alpha_{y}}(2 \pi)^{2}} \int \frac{q d q d \vartheta}{4\left(\left(\delta+q^{2}\right)^{2}+f(\theta) q^{2}+\Delta^{2}\right)^{3 / 2}} .
$$

where $f(\vartheta)=\frac{v^{2} \cos ^{2} \vartheta}{\alpha_{x}}$. Write the integral as

$$
\begin{aligned}
e_{x x} & =-\frac{4 e v \alpha \beta \Delta}{8 \sqrt{\alpha_{x} \alpha_{y}}(2 \pi)^{2}} \int\left[\int \frac{d q^{2}}{\left(\left(\delta+q^{2}\right)^{2}+f(\vartheta) q^{2}+\Delta^{2}\right)^{3 / 2}}\right] d \vartheta \\
& \equiv-\frac{4 e v \alpha \beta \Delta}{8 \sqrt{\alpha_{x} \alpha_{y}}(2 \pi)^{2}} \int g(\vartheta) d \vartheta
\end{aligned}
$$

where

$$
g(\vartheta)=\int \frac{d q^{2}}{\left(\left(\delta+q^{2}\right)^{2}+f(\vartheta) q^{2}+\Delta^{2}\right)^{\frac{3}{2}}}
$$


Since $\frac{f(\vartheta) q^{2}}{\left(\delta+q^{2}\right)^{2}+\Delta^{2}}$ goes to zero for both $q \rightarrow 0$ and $q \rightarrow+\infty$, it may be justified to do a Taylor expansion about it. Then we used Taylor expansion around $\Gamma$ point for Eq. (S15), and obtained

$$
\begin{gathered}
g(\vartheta) \approx \int \frac{1}{\left((\delta)^{2}+\triangle^{2}\right)^{3 / 2}}-\frac{3 f(\vartheta) q^{2}}{2\left((\delta)^{2}+\triangle^{2}\right)^{5 / 2}} d q^{2} \\
g(\vartheta) \approx \frac{q^{2}}{\left((\delta)^{2}+\triangle^{2}\right)^{3 / 2}}-\frac{3 f(\vartheta) q^{4}}{4\left((\delta)^{2}+\triangle^{2}\right)^{5 / 2}}
\end{gathered}
$$

The piezoelectric coefficients $e_{x x}$ (Eq. (9)) can thus be approximated as

$$
\begin{gathered}
e_{x x} \approx-\frac{4 e v \alpha \beta \Delta}{8 \sqrt{\alpha_{x} \alpha_{y}(2 \pi)^{2}}} \int \frac{q^{2}}{\left((\delta)^{2}+\triangle^{2}\right)^{3 / 2}}-\frac{3 f(\vartheta) q^{4}}{4\left((\delta)^{2}+\triangle^{2}\right)^{5 / 2}} d \vartheta \\
e_{x x} \approx-\frac{4 e v \alpha \beta \Delta}{8 \sqrt{\alpha_{x} \alpha_{y}(2 \pi)}}\left(\frac{q^{2}}{\left((\delta)^{2}+\triangle^{2}\right)^{3 / 2}}-\frac{3 q^{4}}{8\left((\delta)^{2}+\triangle^{2}\right)^{5 / 2}} \frac{v^{2}}{\alpha_{x}}\right) \\
e_{x x} \approx-\frac{e v \alpha \beta \Delta}{4(\pi)^{2}}\left(\frac{S_{B Z, q}}{\left((\delta)^{2}+\triangle^{2}\right)^{3 / 2}}-\frac{3 S_{B Z, q}^{2}}{8 \pi\left((\delta)^{2}+\triangle^{2}\right)^{5 / 2}} \frac{v^{2}}{\alpha_{x}}\right) \\
e_{x x} \approx-\frac{e v \alpha \beta \Delta S_{B Z}}{4(\pi)^{2}}\left(\frac{1}{\left((\delta)^{2}+\triangle^{2}\right)^{3 / 2}}-\frac{3 S_{B Z} v^{2}}{8 \pi\left((\delta)^{2}+\triangle^{2}\right)^{5 / 2}} \sqrt{\frac{\alpha_{y}}{\alpha_{x}}}\right) \\
e_{x x} \approx-\frac{c_{1} v \alpha \Delta}{x_{0}{ }^{2}}\left(\frac{1}{\left((\delta)^{2}+\triangle^{2}\right)^{3 / 2}}-\frac{c_{2}}{\left((\delta)^{2}+\triangle^{2}\right)^{5 / 2}} \frac{v^{2}}{x_{0}^{2}} \sqrt{\frac{\alpha_{y}}{\alpha_{x}}}\right)
\end{gathered}
$$

where $v=x_{0}\left(-2 c t_{2}-t_{1}\right)$. For planer and buckled structures $c<1$, and for puckered structure $c>1$.

$$
e_{x x} \approx \frac{c_{1} \Delta \alpha\left(2 c t_{2}+t_{1}\right)}{x_{0}}\left(\frac{1}{\left((\delta)^{2}+\triangle^{2}\right)^{3 / 2}}-\frac{c_{2}\left(2 c t_{2}+t_{1}\right)^{2}}{\left((\delta)^{2}+\triangle^{2}\right)^{5 / 2}} \sqrt{\frac{\alpha_{y}}{\alpha_{x}}}\right)
$$

piezoelectric coefficients of planer, buckled and puckered monolayers can be written in identical form $e_{x x} \approx c_{1} \frac{\Delta}{x_{0}} f(t, \triangle)+c_{2}$. According to the assumptions presented in Table 1, the piezoelectric coefficients of planer and buckled structures are approximated as 


$$
e_{x x} \approx c_{1} \frac{\Delta}{x_{0}} \frac{t^{2}}{\left(t^{2}+\Delta^{2}\right)^{3 / 2}}\left[1-c_{2} \frac{t^{2}}{\left(t^{2}+\Delta^{2}\right)}\right]+c_{3}
$$

while the piezoelectric coefficient of puckered structures is approximated as

$$
e_{x x} \approx c_{1} \frac{\Delta}{x_{0}} \frac{\left(t_{1}+\cos 45^{\circ} t_{2}\right)\left(t_{1}+t_{2} \mathrm{c}\right)}{\left(\left(t_{1}-2 t_{2}\right)^{2}+\Delta^{2}\right)^{\frac{3}{2}}}\left[1-\frac{c_{2}\left(2 c t_{2}+t_{1}\right)^{2}}{\left(t_{1}-2 t_{2}\right)^{2}+\Delta^{2}}\right]+c_{4}
$$

Except for the above common TCH structures, we calculated the piezoelectric coefficients of another special TCH structure (spatial planer) to further illustrate the generality of our model. The atomic structure and the related assumption for tight binding parameters of spatial planer monolayer are summarized in Table S1.

$$
\begin{gathered}
e_{x x} \approx \frac{e}{2 \pi^{2}} \iint \frac{-2 t x_{0} \mathrm{t} \beta \Delta}{\left(\delta^{2}+\left(2 t x_{0} k_{x}\right)^{2}+\left(2 t x_{0} k_{y}\right)^{2}+\triangle^{2}\right)^{3 / 2}} d k_{x} d k_{y} \\
e_{x x} \approx c \int \frac{t x_{0} \mathrm{t} \beta \triangle \rho d \rho}{\left(\delta^{2}+\left(2 t x_{0}\right)^{2} \rho^{2}+\triangle^{2}\right)^{3 / 2}}
\end{gathered}
$$

where $\rho^{2}=k_{x}{ }^{2}+k_{y}{ }^{2}$.

$$
\begin{gathered}
e_{x x} \approx c \frac{\Delta}{x_{0}}\left(\frac{1}{\left(\delta^{2}+\triangle^{2}\right)^{1 / 2}}-\frac{1}{\left(\delta^{2}+c\left(t x_{0}\right)^{2} S_{B Z}+\triangle^{2}\right)^{1 / 2}}\right)+c_{1} \\
e_{x x} \approx c_{1} \frac{\Delta}{x_{0}}\left(\frac{1}{\left(t^{2}+\triangle^{2}\right)^{1 / 2}}-\frac{1}{\left(t^{2}+c t^{2}+\triangle^{2}\right)^{1 / 2}}\right)+c_{2}
\end{gathered}
$$

Table S1. Structural characters and piezoelectric coefficient of spatial planer monolayer.

Structure

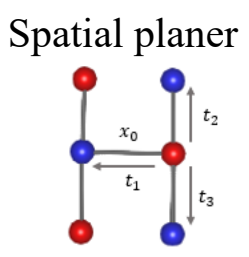

Assumptions

$$
\begin{gathered}
t_{1}=t_{2}=t \\
\theta=90^{\circ} \\
l_{x}=x_{0} \\
l_{y}=x_{0}
\end{gathered}
$$

Results 


\section{E. Orbital hybridization}

As an application of the theoretical model, we probed the origin of large difference in piezoelectricity between $\mathrm{GeS}$ and GeSe monolayers. It turns out that both monolayers have comparable bandgaps from the projected density of states (PDOS) computed by DFT approach as shown in Figure S2. However, the GeS monolayer possesses larger orbital hybridization between Ge and Se atoms around the Fermi level than that of GeSe monolayer, resulting in lower piezoelectricity as expected from the theoretical model.

(a)

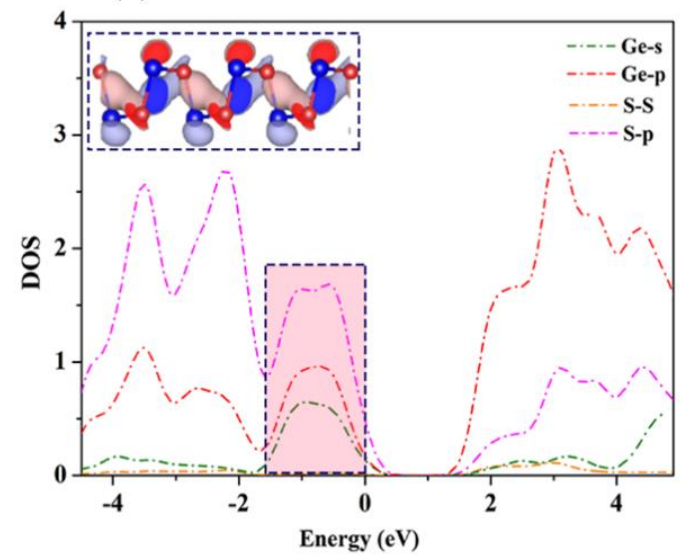

(b)

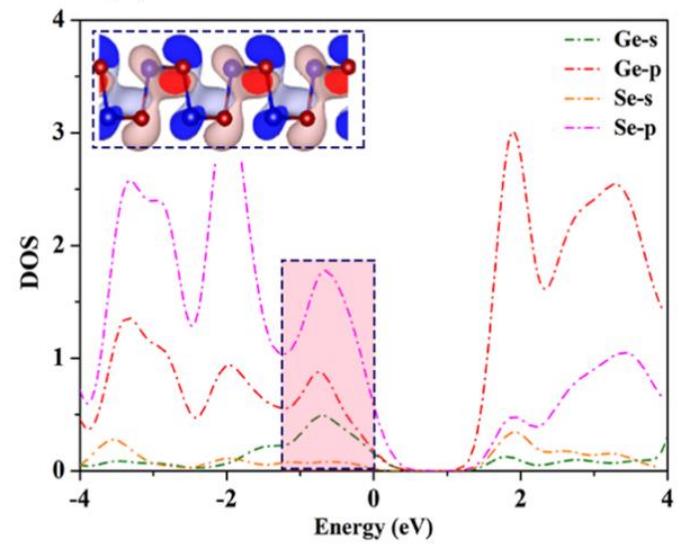

Figure S2. Projected density of states (PDOS) of (a) GeS monolayer and (b) GeSe monolayer.

Relevant orbitals in our tight binding model are highlighted by pink rectangle.

\section{F. Convex hull}

Convex hull energy has been demonstrated to be an effective criterion for probing compound stability. The concept is illustrated by the convex hull diagram of $\mathrm{SnS}$ monolayer as shown in Figure S3. The SnS monolayer is regarded as stable if its formation energy lies in the light blue area. In case that its formation energy lies in the pink area, the distance between the formation energy and the boundary line connecting existent compounds (black line) is 
further checked. The SnS monolayer is considered to be stable if the distance is smaller than 0.2 .

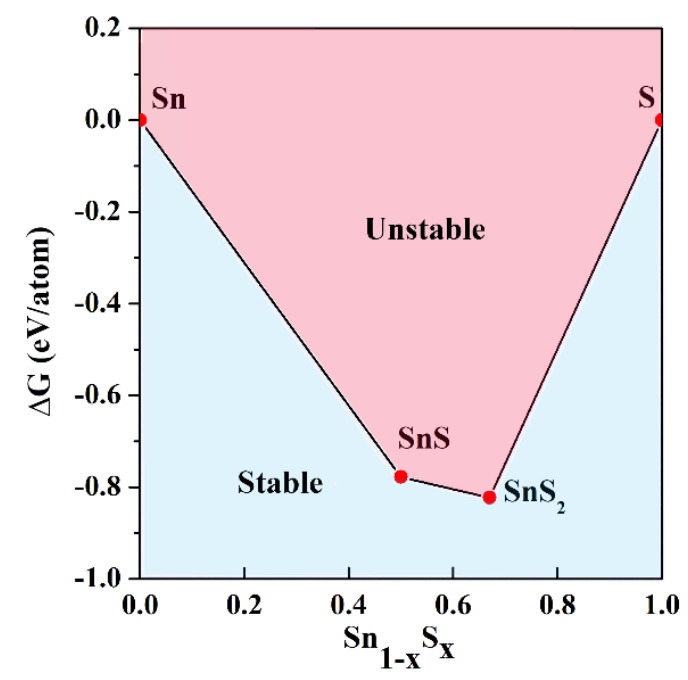

Figure S3. Convex hull diagram for $\mathrm{SnS}$ monolayer. The formation energies of existent compounds are denoted by red dots.

\section{G. DFT results}

The results from DFT calculations are summarized in Table S2 S4. The piezoelectric coefficients obtained in this study are consistent with those reported by previous works. ${ }^{3}$ The predictive model for infrared materials is presented in Eq. (16a) of main text. As an exception, the piezoelectric coefficient of $\mathrm{BN}$ monolayer is derived based on its ultraviolet bandgap character as shown in Eq. (S30). The fitting coefficients $c_{1}$ and $c_{2}$ are identical to those of other planer structures, i.e. $c_{1}=2.73$ and $c_{2}=0.16$.

$$
e_{x x} \approx c_{1} \frac{E_{M}}{x_{0} \times E_{g}}+c_{2}
$$

Table S2 Lattice constant $\left(a_{1}\right.$ and $\left.a_{2}\right)$, axle atomic distance $\left(x_{0}\right)$, band gap $\left(E_{g}\right)$, effective mass $\left(E_{M}\right)$ and piezoelectric coefficient $e_{x x}$ obtained by DFT simulations for planer structures. 


\begin{tabular}{|l|l|l|l|l|l|l|}
\hline & \multicolumn{1}{|c|}{$a_{1}$} & $a_{2}$ & $x_{0}$ & $E_{g}$ & $E_{M}\left(\mathrm{~m}_{\mathrm{e}}\right)$ & $e_{x x}(\mathrm{DFT})$ \\
\hline $\mathrm{BP}$ & 3.212 & 3.212 & 1.851 & 1.891 & 1.098 & 2.199 \\
\hline $\mathrm{AlN}$ & 3.123 & 3.123 & 1.802 & 3.034 & 0.924 & 2.169 \\
\hline $\mathrm{BN}$ & 2.490 & 2.490 & 1.437 & 4.892 & 2.079 & 1.235 \\
\hline $\mathrm{GaN}$ & 3.222 & 3.222 & 1.862 & 2.278 & 0.572 & 2.716 \\
\hline $\mathrm{PbS}$ & 4.799 & 4.799 & 2.773 & 1.081 & 1.204 & 0.707 \\
\hline $\mathrm{AlP}$ & 3.938 & 3.938 & 2.269 & 2.460 & 0.469 & 1.147 \\
\hline $\mathrm{AlSb}$ & 4.460 & 4.460 & 2.572 & 0.726 & 0.221 & 0.151 \\
\hline
\end{tabular}

Table S3 Lattice constant ( $a_{1}$ and $a_{2}$ ), axle atomic distance $\left(x_{0}\right)$, band gap $\left(E_{g}\right)$, effective mass $\left(E_{M}\right)$ and piezoelectric coefficient $e_{x x}$ obtained by DFT simulations for buckled structures.

\begin{tabular}{|l|l|l|l|l|l|l|}
\hline & \multicolumn{1}{|c|}{$a_{1}$} & \multicolumn{1}{|c|}{$a_{2}$} & \multicolumn{1}{|c|}{$x_{0}$} & $E_{g}$ & $E_{M}\left(\mathrm{~m}_{\mathrm{e}}\right)$ & $e_{x x}(\mathrm{DFT})$ \\
\hline $\mathrm{AlAs}$ & 4.059 & 4.059 & 2.341 & 1.831 & 0.341 & 0.666 \\
\hline $\mathrm{AsSb}$ & 3.867 & 3.867 & 2.234 & 1.475 & 0.275 & 0.720 \\
\hline $\mathrm{BAs}$ & 3.390 & 3.390 & 1.960 & 1.790 & 0.609 & 1.607 \\
\hline $\mathrm{BiSb}$ & 4.245 & 4.245 & 2.446 & 0.954 & 0.227 & 0.385 \\
\hline $\mathrm{CO}$ & 2.353 & 2.353 & 1.355 & 3.511 & 1.054 & 7.640 \\
\hline $\mathrm{GaAs}$ & 4.051 & 4.051 & 2.334 & 1.244 & 0.217 & 0.390 \\
\hline $\mathrm{GaP}$ & 3.916 & 3.916 & 2.260 & 1.803 & 0.290 & 0.421 \\
\hline $\mathrm{GaSb}$ & 4.379 & 4.379 & 2.524 & 0.858 & 0.184 & 0.468 \\
\hline
\end{tabular}




\begin{tabular}{|c|c|c|c|c|c|c|}
\hline $\mathrm{GeO}$ & 3.019 & 3.019 & 1.743 & 2.241 & 0.887 & 4.886 \\
\hline GeS & 3.497 & 3.497 & 2.024 & 2.485 & 0.585 & 3.103 \\
\hline GeSe & 3.673 & 3.673 & 2.122 & 2.284 & 0.423 & 1.643 \\
\hline InAs & 4.379 & 4.379 & 2.531 & 0.795 & 0.217 & 0.834 \\
\hline InN & 3.634 & 3.634 & 2.099 & 0.482 & 0.299 & 2.047 \\
\hline $\mathrm{InP}$ & 4.245 & 4.245 & 2.452 & 1.176 & 0.259 & 0.318 \\
\hline $\mathrm{InSb}$ & 4.680 & 4.680 & 2.703 & 0.693 & 0.190 & 0.144 \\
\hline $\mathrm{PbO}$ & 3.597 & 3.597 & 2.074 & 1.825 & 1.061 & 4.967 \\
\hline $\mathrm{PbSe}$ & 4.095 & 4.095 & 2.366 & 1.857 & 0.491 & 2.041 \\
\hline $\mathrm{PbTe}$ & 4.347 & 4.347 & 2.505 & 1.625 & 0.417 & 2.573 \\
\hline $\mathrm{PN}$ & 2.722 & 2.722 & 1.568 & 1.837 & 0.828 & 3.513 \\
\hline $\mathrm{SbAs}$ & 3.867 & 3.867 & 2.234 & 1.475 & 0.275 & 0.669 \\
\hline $\mathrm{SbBi}$ & 4.245 & 4.245 & 2.446 & 0.954 & 0.227 & 0.386 \\
\hline SiS & 3.304 & 3.304 & 1.905 & 2.221 & 0.642 & 2.199 \\
\hline $\mathrm{SiSe}$ & 3.527 & 3.527 & 2.037 & 2.135 & 0.431 & 2.169 \\
\hline $\mathrm{SnO}$ & 3.344 & 3.344 & 1.929 & 1.833 & 0.935 & 4.529 \\
\hline $\mathrm{SnSe}$ & 3.914 & 3.914 & 2.261 & 2.220 & 0.464 & 2.716 \\
\hline $\mathrm{SnTe}$ & 4.192 & 4.192 & 2.419 & 1.906 & 0.329 & 0.707 \\
\hline TiS & 3.526 & 3.526 & 2.031 & 0.527 & 8.595 & 12.507 \\
\hline TiSe & 3.665 & 3.665 & 2.112 & 0.400 & 12.743 & 14.324 \\
\hline TiTe & 3.922 & 3.922 & 2.265 & 0.222 & 13.090 & 9.802 \\
\hline
\end{tabular}

Table S4 Lattice constant ( $a_{1}$ and $a_{2}$ ), axle atomic distance $\left(x_{0}\right)$, band gap $\left(E_{g}\right)$, effective mass $\left(E_{M}\right)$ and piezoelectric coefficient $e_{x x}$ obtained by DFT simulations for puckered structures. 


\begin{tabular}{|l|l|l|l|l|l|l|}
\hline & \multicolumn{1}{|c|}{$a_{1}$} & \multicolumn{1}{|c|}{$a_{2}$} & $x_{0}$ & $E_{g}$ & $E_{M}\left(\mathrm{~m}_{\mathrm{e}}\right)$ & $e_{x x}(\mathrm{DFT})$ \\
\hline $\mathrm{GeO}$ & 2.985 & 3.741 & 0.378 & 2.894 & 1.235 & 2.869 \\
\hline $\mathrm{GeS}$ & 3.670 & 4.434 & 0.382 & 1.779 & 1.878 & 3.124 \\
\hline $\mathrm{GeSe}$ & 3.981 & 4.276 & 0.148 & 1.143 & 11.605 & 8.412 \\
\hline $\mathrm{GeTe}$ & 4.240 & 4.375 & 0.068 & 0.855 & 3.312 & 7.317 \\
\hline $\mathrm{NBi}$ & 3.436 & 3.502 & 0.033 & 1.309 & 2.538 & 12.148 \\
\hline $\mathrm{NSb}$ & 3.287 & 3.562 & 0.137 & 1.643 & 2.600 & 3.790 \\
\hline $\mathrm{PAs}$ & 3.505 & 4.709 & 0.602 & 0.929 & 1.484 & 2.915 \\
\hline $\mathrm{PBi}$ & 4.118 & 4.456 & 0.169 & 0.741 & 3.544 & 1.161 \\
\hline $\mathrm{SbP}$ & 3.906 & 4.461 & 0.278 & 0.495 & 2.868 & 1.460 \\
\hline $\mathrm{SiS}$ & 3.345 & 4.809 & 0.732 & 1.431 & 1.428 & 5.435 \\
\hline $\mathrm{SiSe}$ & 3.599 & 4.778 & 0.589 & 1.193 & 1.786 & 6.682 \\
\hline $\mathrm{SiTe}$ & 4.115 & 4.275 & 0.080 & 0.397 & 3.054 & 7.703 \\
\hline $\mathrm{SnO}$ & 3.246 & 3.331 & 0.042 & 2.345 & 1.896 & 9.573 \\
\hline $\mathrm{SnS}$ & 4.077 & 4.242 & 0.083 & 1.471 & 2.148 & 7.973 \\
\hline $\mathrm{SnSe}$ & 4.302 & 4.359 & 0.029 & 0.903 & 7.949 & 23.955 \\
\hline $\mathrm{SnTe}$ & 4.559 & 4.579 & 0.011 & 0.727 & 4.373 & 44.266 \\
\hline
\end{tabular}

\section{H. Correlation analysis}

Despite the negative correlation coefficients between $e_{x x}$ and $E_{g}$, both $E_{M} \times \sqrt{E_{g}}$ and $E_{M} / \sqrt{E_{g}}$ have smaller correlation coefficients than $E_{M}$. This can be explained by the distinct 
expressions of piezoelectricity for different types of TCH materials. Specifically, $E_{M} \times \sqrt{E_{g}}$ possesses larger correlation coefficient for planer and buckled structures (Figure S4a) while smaller correlation coefficient (Figure S4b) compared with $E_{M} / \sqrt{E_{g}}$.

(a)

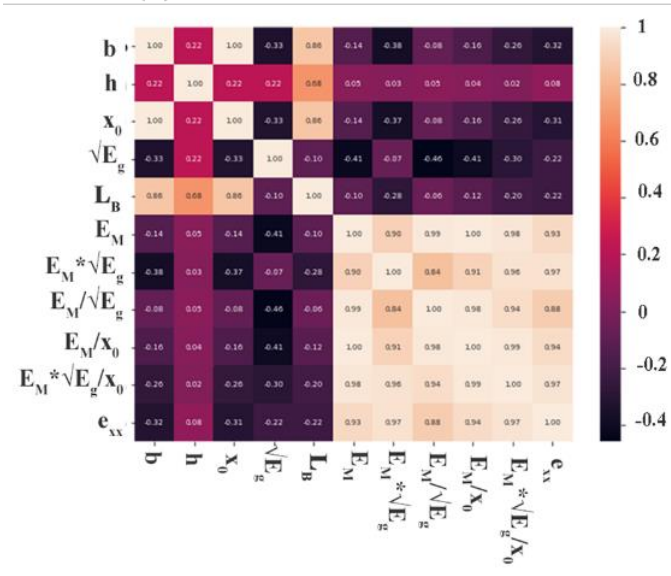

(b)

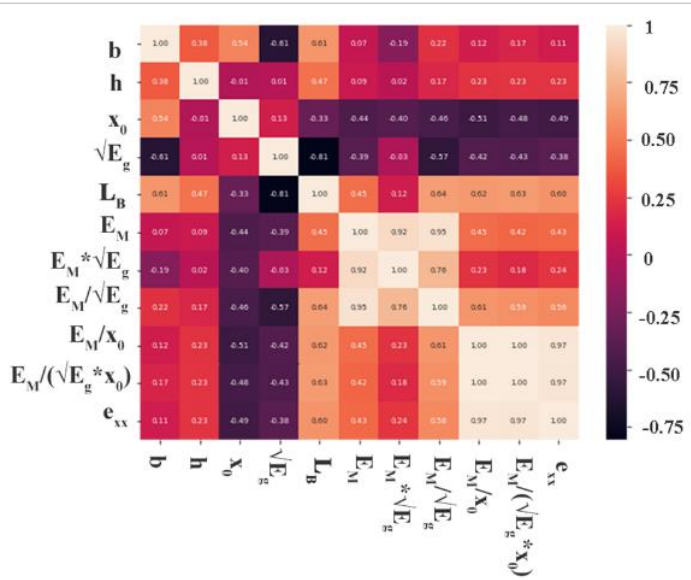

Figure S4. Correlation analyses between piezoelectricity and related parameters for (a) buckled and planer monolayers and (b) puckered monolayers.

\section{Cross-validation}

Cross validation (LFCV) is a data enhancement approach within the category of machine learning that recurrently utilizes subsets of data with different roles. For each group of buckled, planer and puckered monolayers, the data is randomly split into five sub-groups in LFCV process. Linear fitting (LF) is then conducted for five times, with the five sub-groups taking turns to serve as test set and the remaining four sub-groups serving as training set. The linear correlation constants $c_{1}$ and $c_{2}$ are calculated by averaging over the results of five fittings. The comparisons between the results obtained by the original linear fitting method and the LFCV method are summarized in Table S5 and Figure S5. It turns out that the linear correlation 
constants $c_{1}$ and $c_{2}$ attained by both approaches are comparable to each other. The Rsquared score of the LFCV method is slightly higher than that of the LF method for the puckered monolayers, while equal R-square scores are observed for both the buckled and planer monolayers. In all, the performance of the LF almost reaches that of the LFCV method as visualized in Figure S5. This demonstrates the reliability of our model and justifies the results obtained by the LF method training with the entire data set.

Table S5 Comparison of fitting coefficients and R-squared scores obtained by the LF and LFCV methods.

\begin{tabular}{|c|c|c|c|c|}
\hline Structures & \multicolumn{2}{|c|}{ LF } & \multicolumn{2}{c|}{ LFCV } \\
\hline Buckled & $c_{1}=4.03 ; c_{2}=0.53$ & $\mathrm{R}^{2}=0.95$ & $c_{1}=4.02 ; c_{2}=0.59$ & $\mathrm{R}^{2}=0.95$ \\
\hline Planer & $c_{1}=2.73 ; c_{2}=0.16$ & $\mathrm{R}^{2}=0.96$ & $c_{1}=2.70 ; c_{2}=0.19$ & $\mathrm{R}^{2}=0.96$ \\
\hline Puckered & $c_{1}=0.08 ; c_{2}=3.23$ & $\mathrm{R}^{2}=0.94$ & $c_{1}=0.08 ; c_{2}=3.58$ & $\mathrm{R}^{2}=0.95$ \\
\hline
\end{tabular}
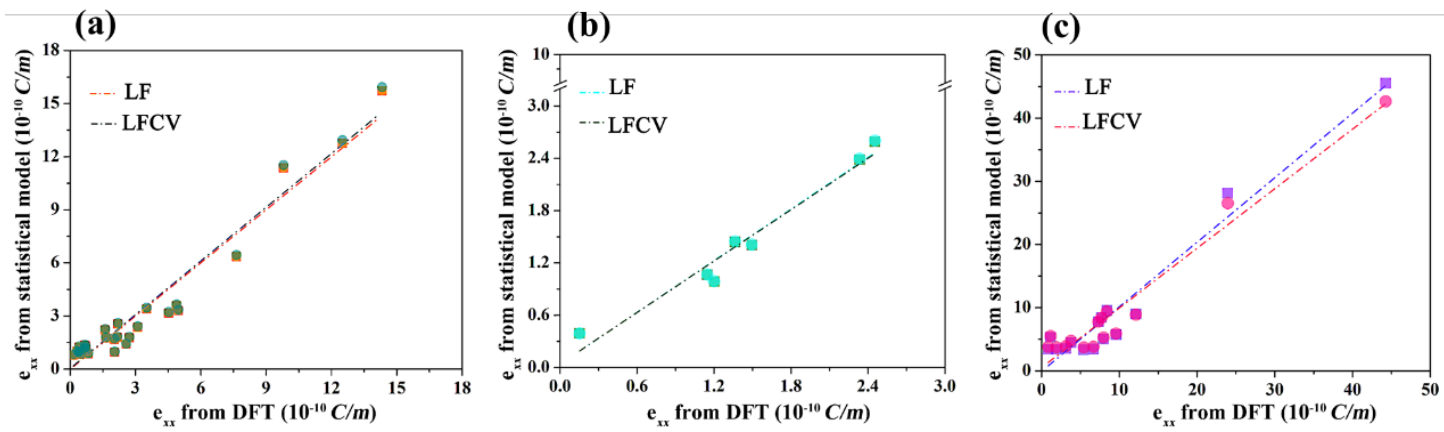

Figure S5. Reliability check of piezoelectric model. Comparison of results obtained by LF and LFCV methods for (a) buckled, (b) planer and (c) puckered monolayers. 


\section{J. Analysis of effective mass}

We considered the dependence of effective mass on theoretical variables $t$ and $\Delta$. The effective mass increases with $\Delta$, while decreases with increasing $t$ as shown in Figure S6.

(a)

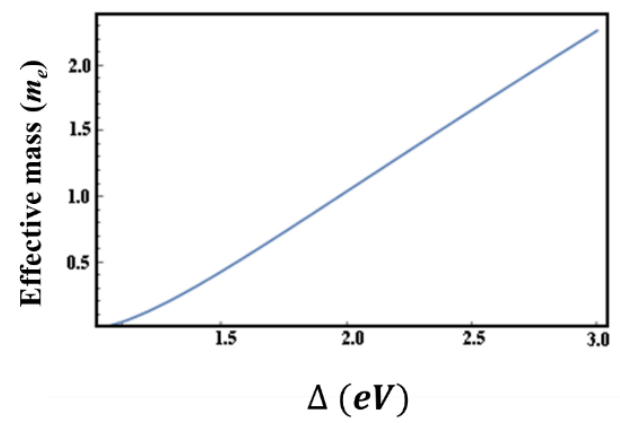

(b)

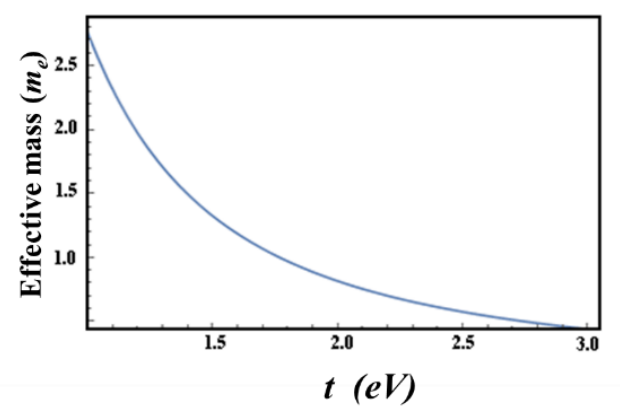

Figure S6. Dependence of effective mass on (a) $\Delta$ and (b) $t$.

\section{References}

1. Castro Neto, A. H.; Guinea, F.; Peres, N. M. R.; Novoselov, K. S.; Geim, A. K. The Electronic Properties of Graphene. Rev. Mod. Phys. 2009, 81, 109-162.

2. Naumis, G. G.; Barraza-Lopez, S.; Oliva-Leyva, M.; Terrones, H. Electronic and Optical Properties of Strained Graphene and Other Strained 2D Materials: a Review. Rep. Prog. Phys. 2017, 80, 096501.

3. Blonsky, M. N.; Zhuang, H. L.; Singh, A. K.; Hennig, R. G. Ab Initio Prediction of Piezoelectricity in Two-Dimensional Materials. ACS nano 2015, 9 , 9885-9891. 